NASA Technical Memorandum 101295

\title{
Active Phase Compensation System for Fiber Optic Holography
}

Carolyn R. Mercer and Glenn Beheim

Lewis Research Center

Cleveland, Ohio

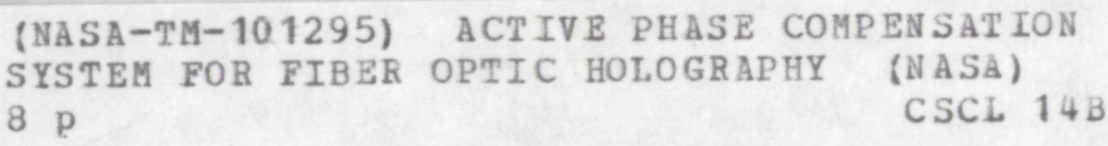

Prepared for the

32nd Annual International Technical Symposium on Optical and Optoelectronic Applied Science and Engineering sponsored by the Society of Photo-Optical Instrumentation Engineers San Diego, California, August 14-19, 1988 


\section{Active phase compensation system for fiber optic holography}

Carolyn R. Mercer and Glenn Beheim

NASA Lewis Research Center, Cleveland, Ohio 44135

\section{ABSTRACT}

Fiber optic delivery systems promise to extend the application of holography to severe environments by simplifying test configurations and permitting the laser to be remotely placed in a more benign location. However, the introduction of optical fiber leads to phase stability problems. Environmental effects cause the pathlengths of the fibers to change randomly, preventing the formation of stationary interference patterns which are required for holography. An active phase control system has been designed and used with an all-fiber optical system to stabilize the phase difference between light emitted from two fibers, and to step the phase difference by $90^{\circ}$ without applying any constraints on the placement of the fibers. The accuracy of the phase steps is shown to be better than $0.02^{\circ}$, and a stable phase difference can be maintained for $30 \mathrm{~min}$. This system can be applied to both conventional and electro-optic holography, as well as to any system where the maintenance of an accurate phase difference between two coherent beams is required.

\section{INTRODUCTION}

Accurate control of the relative phase between two coherent beams is required in many interferometric instruments. Conventional holographic and interferometric systems require two beams to have a fixed phase difference for times ranging from microseconds to hours. Electro-optic holography, a measurement method of considerable interest in which holographic interferograms are recorded on relatively coarse electronic arrays, requires the relative phase between the two beams to be sequentially stepped. 1 A system is described here which allows the phase difference between two coherent beams to be accurately stabilized, and to be automatically stepped through a sequence of four $90^{\circ}$ phase increments. It is based on a system developed for phase stabilization by Neumann and Rose, 2 and on a fiber optic adaptation by Corke, et al:3

We have chosen to use an all-fiber optical system because of the advantages inherent in using fibers, such as ease of alignment, a minimal number of required optical components, and the ability to locate the laser away from the test area. Working with $f$ ibers instead of conventional optics allows changes in optical configurations to be made quickly and simply, without the need for realigning sensitive components. The use of single mode fibers eliminates the need for lens/pinhole spatial filters. Optical fibers can be used as light pipes to safely deliver optical power to noisy environments where a laser may not work properly, or to distribute light from a single laser to several locations. The principle disadvantage of using fibers in holographic systems is the high sensitivity of the phase of the output light to slight variations in the fiber temperature and to small mechanical stresses induced within the fiber. Even in a benign laboratory environment, holograms can not be reliably recorded using fibers for light delivery when the exposure times are longer than about $100 \mathrm{~ms}$; active phase compensation must be used. This paper describes a phase compensation system that provides phase stabilization as well as phase stepping. The underlying theory of the system is described, followed by details on the electronics and the performance of the system.

\section{PRINCIPLE OF OPERATION}

The optical configuration is shown in Fig. 1. Light from a $25 \mathrm{~mW}$ Helium-Neon laser is coupled into one arm (Fl) of a single-mode fiber optic coupler. The coupler has a splitting ratio of $90 / 10$, which is useful for holography where the object beam must be substantially brighter than the reference beam; however, this system works independently of the coupling ratio chosen. The objective is to stabilize the relative phase, $\theta$, between the light emitted from the two output fibers F2 and F3. In order to monitor $\theta$ for use in the phase control circuit, a photodiode, PDl, is used to detect the light reflected back from the ends of the output fibers F2 and F3 which interferes in the coupler arm F4. The amplitude of this detected signal varies as a function of $2 \theta$ due to the double-pass of the light through the output fibers. For demonstration of our phase control system, a beamsplitter was used to combine the light emitted from the output fiber ends to permit direct measurement of $\theta$ by a second photodetector, PD2. Note that phase disturbances caused by motion of components that intersect the optical path after the light has left the fibers can not be detected by PDI. 
Approximately $5 \mathrm{~m}$ of each of the output fibers are wrapped tightly around a piezoelectric transducer (PZTl and PZT2) to provide pathlength control of both output fibers. $A$ sinusoidal voltage is applied to PZT1 so that PDI senses a phase-modulated signal described by

$$
I(t)=I_{0}\{1+\eta \cos [2 \theta-2 \beta \sin (\omega t)]\}
$$

The frequency and amplitude of the dither signal are represented by $\omega$ and $\beta$, respectively, $n$ is the unmodulated interference fringe visibility, and $\theta$ is the mean phase difference between the light at the output faces of fibers F2 and F3. The Fourier expansion of this function is given by

$$
\begin{gathered}
I(t)_{0}=I_{0}+n I_{0} \cos (2 \theta)\left[J_{0}(2 \beta)+\sum_{n} 2 J_{n}(2 \beta) \sin (n \omega t)\right] \\
\quad \therefore \quad+n I_{0} \sin (2 \theta)\left[\sum_{n} 2 J_{n}(2 \beta) \sin (n \omega t)\right]
\end{gathered}
$$

where $J_{n}$ represents the $n^{\text {th }}$ order Bessel function.

A phase-indicating signal can be obtained by multiplying the photodiode output by $\sin (\omega t)$ and low pass filtering. This signal is expressed as

$$
\mathrm{v}_{1}=\mathrm{K} \sin (2 \theta)
$$

where $K=\eta I_{0} J_{1}(2 \beta)$.

This: signal can be used as the error signal for a feedback loop that adjusts the voltage applied to PZT2 in order to null: $V_{1}$. By manipulating the sign of the photodiode output one can cause $2 \theta$ to equal either $2 n\left(180^{\circ}\right)$ or $(2 n+1)$ ( $\left.180^{\circ}\right)$ where n is an integer. Phase steps of $180^{\circ}$ at PDl correspond to the desired result of $90^{\circ}$ phase steps at the ends of the output fibers. However, a complication arises due to an ambiguity in the value of $\theta$; for a given phase shift $2 \theta$ at PD1, there are two possible phase shifts at PD2, $\theta$ and $\theta+180^{\circ}$. This ambiguity can be resolved by forcing $\theta$ to be nearly equal to the desired value before activating the phase control circuit.

\section{DESCRIPTION OF ELECTRONICS}

The phase control circuit is implemented with the system shown in Fig. 2 . A $10 \mathrm{kHz}$ oscillator drives PZTI in order to produce the phase modulation signal at PDI described by Eq. 1. The lock-in amplifier multiplies this signal by the oscillator signal, producing $V_{1}$ (Eg. 3).: This error signal is integrated and amplified by a high voltage amplifier with a $1000 \mathrm{~V}$ range, then sent to PZT2, completing the feedback loop.

In order to produce a monotonically increasing sequence of $90^{\circ}$ phase steps, voltage steps are applied to PZT1 which change the phase at PD2 by approximately $90^{\circ}$. While the PZTl bias voltage is being incremented, the phase controller is temporarily inactivated. When reactivated, the controller quickly provides the fine tuning that is required to produce an accurate $90^{\circ}$ phase step, and then holds this phase for the duration of the exposure.

This process was operated under computer control via an IEEE-488 bus. A complete phase stepping sequence consists of the following steps:

1. The integrator is momentarily reset (SWI).

2. The integrator is frozen at its current value (SW2).

3. The sign of the oscillator signal to PZTl is inverted (SW3).

4. The level of the bias voltage to. PZT1 is incremented by $1.05 \mathrm{~V}$ (D/A).

5. The integrator is reactivated to allow integration of the error signal $V_{1}$ (sw2).

A hologram is recorded after step 5, then steps 2 to 5 are repeated for as many phase increments as are necessary. 


\section{PERFORMANCE}

The need for active phase stabilization of a fiber optic based interferometer is shown by the plot of the intensity at PDI as a function of time with and without phase stabilization (Fig. 3). Although the fibers were mounted on a vibration-isolation table, the first half of the plot shows the intensity level rapidly changing due to random phase variations. Activating the phase stabilization circuit causes the intensity to remain constant as is shown in the second half of the plot. The stabilized intensity corresponds to a $180^{\circ}$ relative phase shift at PDI. An ambiguity of $180^{\circ}$ exists at PD2 upon start-up; this should not be a problem provided the system remains active during the course of the experiment.

Figure 4 shows the output at PD2 for five $90^{\circ}$ steps when the initial phase was nearly $0^{\circ}$. The maximum intensity represents constructive interference at $0^{\circ}$ phase difference, the minimum intensity represents destructive interference at $180^{\circ}$, and the intermediate intensities occur at $90^{\circ}$ and $270^{\circ}$. The $90^{\circ}$. and $270^{\circ}$ intensities are unequal due to the nonzero initial phase. The photodetector signal plotted in Fig. 4 was low-pass filtered to remove fluctuations caused by laser intensity noise. The time constant of this filter was l sec which produced the slow response of the measured intensity to the relatively fast phase changes. The phase steps were measured to occur in less than $20 \mathrm{msec}$.

The accuracy of the phase steps was determined by monitoring the error signal $V_{1}$. With the phase compensation circuit inactive, the maximum value of $V_{1}$ was used to infer the magnitude of $K$. With the phase compensation circuit active,- the phase error is given by

$$
\theta_{\text {err }}=\left(\frac{1}{2}\right) \sin ^{-1}\left(\frac{V_{1}}{K}\right)
$$

The phase error $\theta_{\text {err }}$ is plotted as a function of time in Fig. 5, where $\theta_{\text {err }}$ is low-pass filtered with a 1 sec time constant. The maximum phase error is $0.06^{\circ}$, the rms error is $0.02^{\circ}$.

A dither signal amplitude of $0.14 \mathrm{~V}$ caused the phase oscillation amplitude $\beta$ to equal $12^{\circ}$. From Eq. 2, the maximum fringe visibility at PD2 is $J_{0}(\beta) \eta$, or, for $\beta=12^{\circ}$, $0.99 \mathrm{n}$, where $\eta$ is the fringe visibility for the unmodulated case. The fringe visibility is therefore only slightly degraded by the actions of the phase control system.

The frequency response of the phase compensation system is flat to $150 \mathrm{~Hz}$. This was found to be adequate for use in a laboratory environment.

The maximum phase drift that can be compensated before saturating the high voltage amplifier is $28,000^{\circ}$ for our system. After a reset, the high-voltage amplifier remains unsaturated for periods of approximately $1 / 2 \mathrm{hr}$. This range can be extended by wrapping more fiber around PZT2 or by using a high-voltage amplifier with a larger range.

\section{CONCLUSION}

A new way to provide accurate and stable phase stepping has been developed using an all-fiber optical system. This method has been shown to provide a stable phase difference between the outputs of two single mode optical fibers for $30 \mathrm{~min}$ in a laboratory environment. Sequential phase steps of $90^{\circ}$ have been shown to be accurate to within $0.02^{\circ}$. The system compensates for environmentally induced phase drifts of up to $28,000^{\circ}$ in any one direction, with a cut-off frequency of $150 \mathrm{~Hz}$. This system can be used in $f$ iber-optic holography to provide the necessary phase stability between the object and reference beams for exposures of up to $1 / 2 \mathrm{hr}$. Additionally, this system can accurately provide $90^{\circ}$ phase steps for electro-optic holography. The entire system has been automated to allow for synchronization of the phase steps to external data acquisition systems.

\section{REFERENCES}

1. K.A. Stetson and W.R. Brohinsky, "Electrooptic Holography and its Application to Hologram Interferometry," Appl. Opt. 24(21), 3631-3637 (1985).

2. D.B. Neumann and H.W. Rose, "Improvement of Recorded Holographic Fringes by Feedback Control." Appl. Opt. 6(6), 1097-1104 (1967).

3. M. Corke, J.D.C. Jones, A.D. Kersey, and D.A. Jackson, "All Single-Mode Fibre Optic Holographic System with Active Fringe Stabilization," J. Phys. E. 18(3), 185-186 (1985).

4. Y. Ohtsuka, "Dynamic Measurements of Small Displacements by Laser Interferometry," Trans. Inst. Meas. Control, 4(3), 115-124 (1982). 


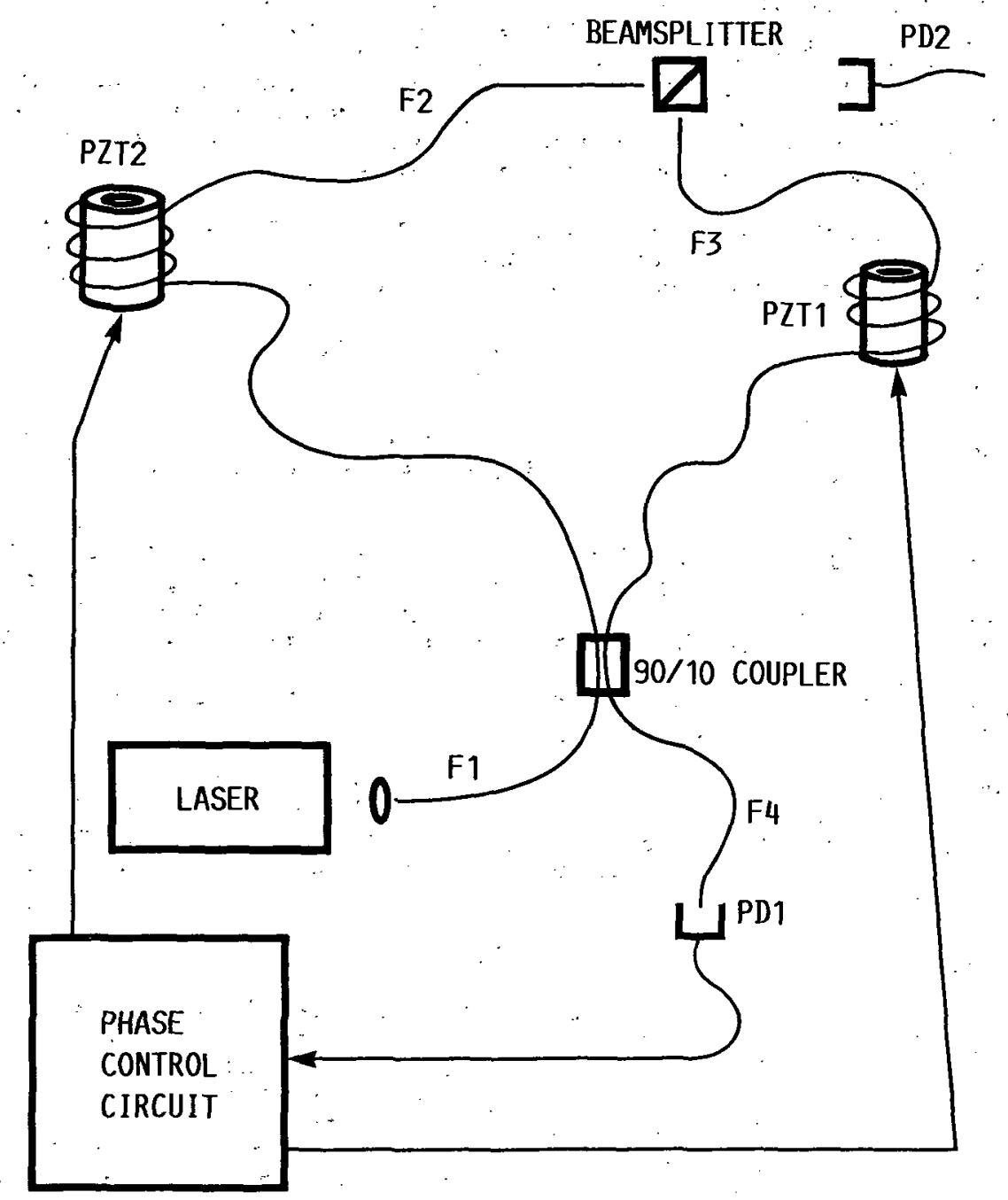

FIGURE 1. - OPTICAL CONFIGURATION FOR PHASE CONTROL SYSTEM. 


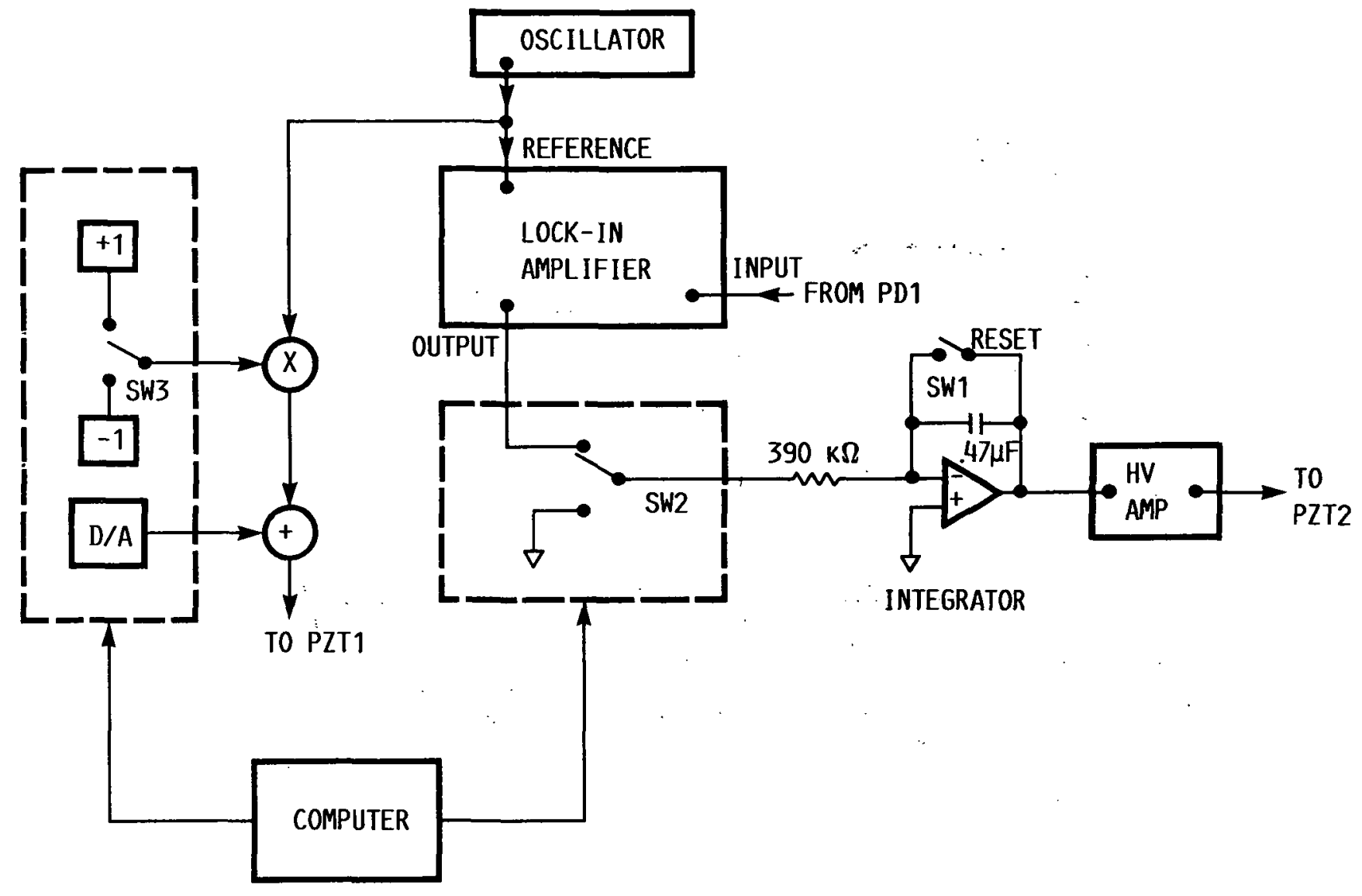

FIGURE 2.- PHASE CONTROL CIRCUIT.

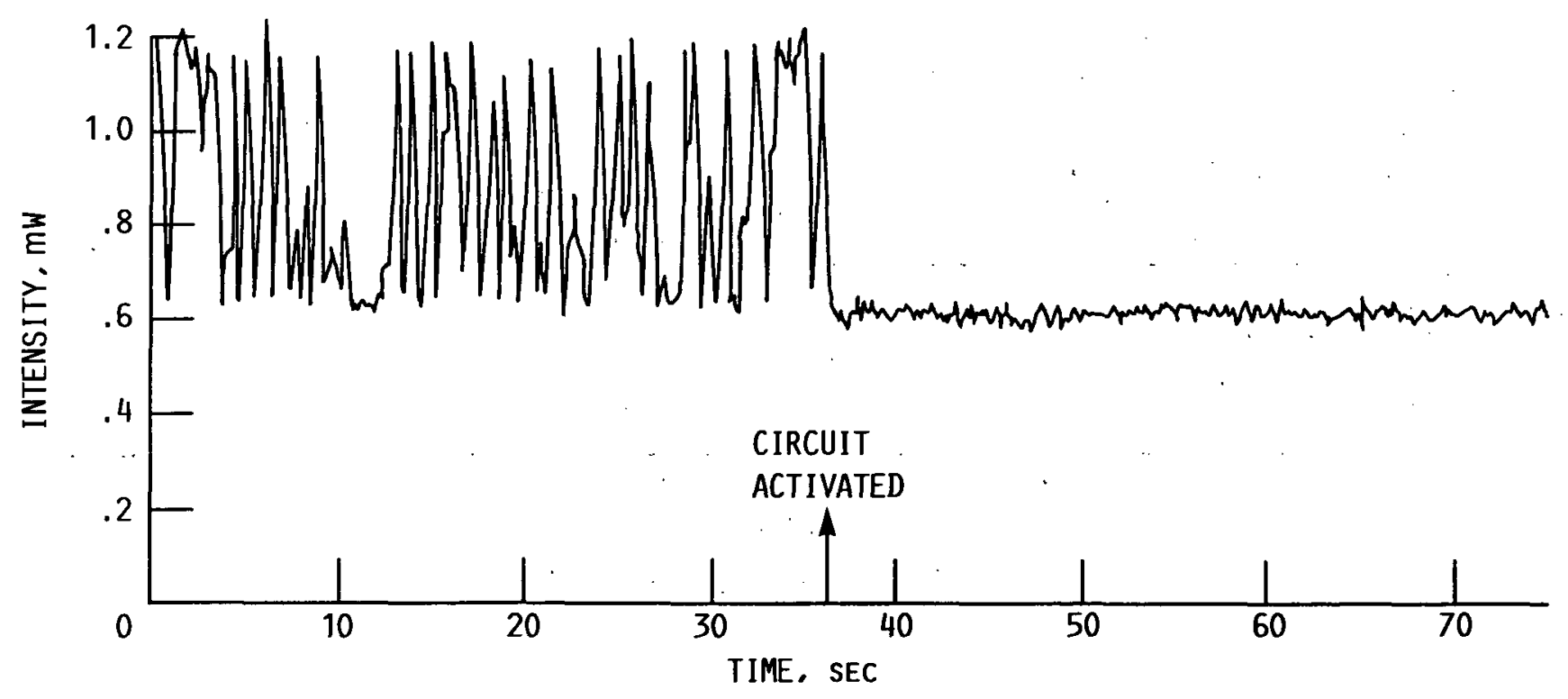

FIGURE 3. - EFFECT OF PHASE STABILIZATION CIRCUIT ON PD1 SIGNAL. 


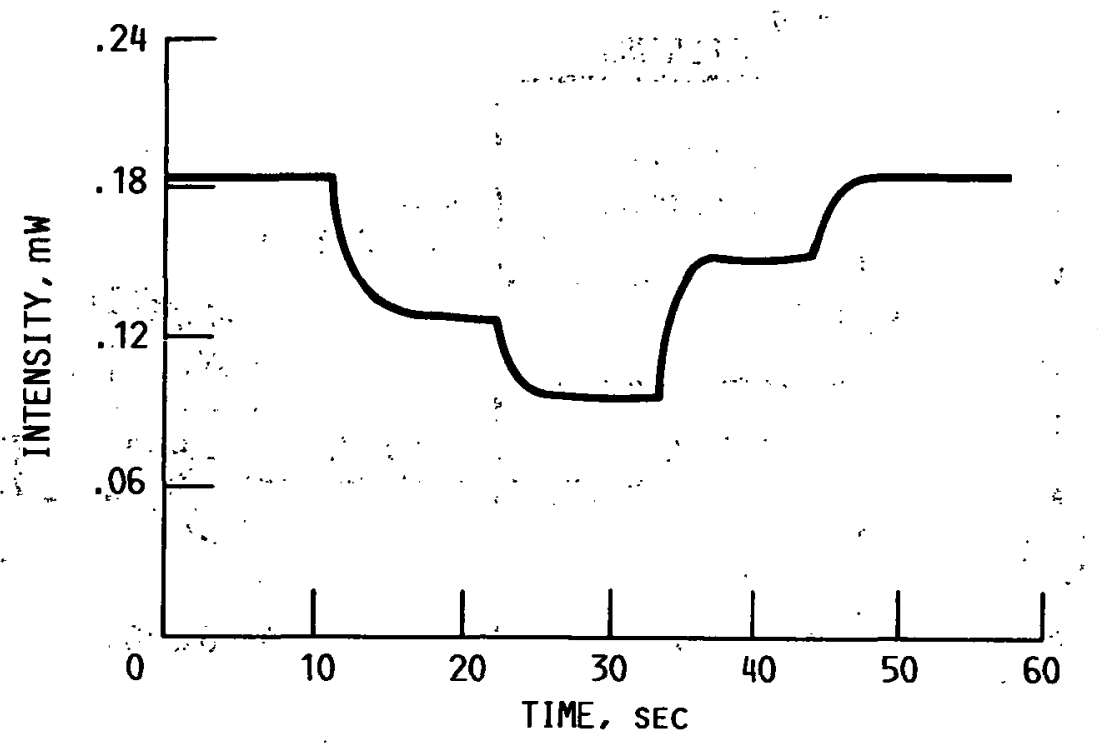

FIGURE 4. - PHOTODETECTOR PD2 SIGNAL FOR FIVE $90^{\circ}$ PHASE STEPS.

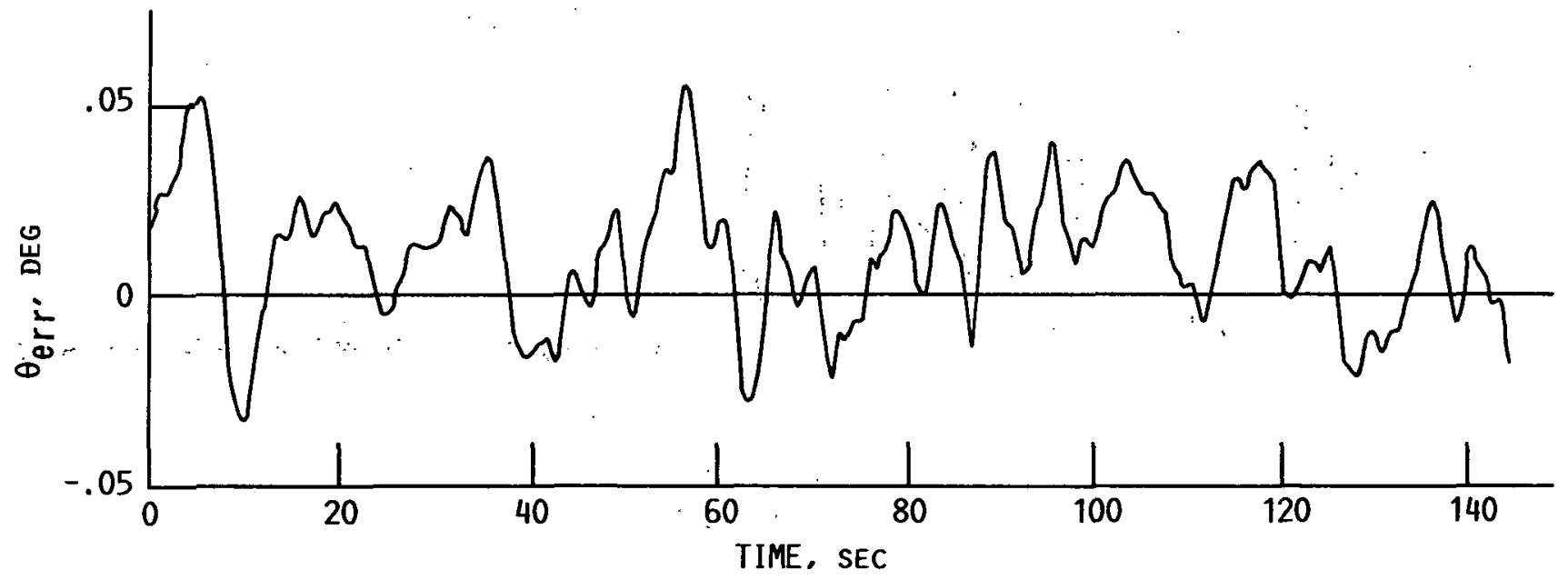

FIGURE 5. - PHASE ERROR AT PD2. 


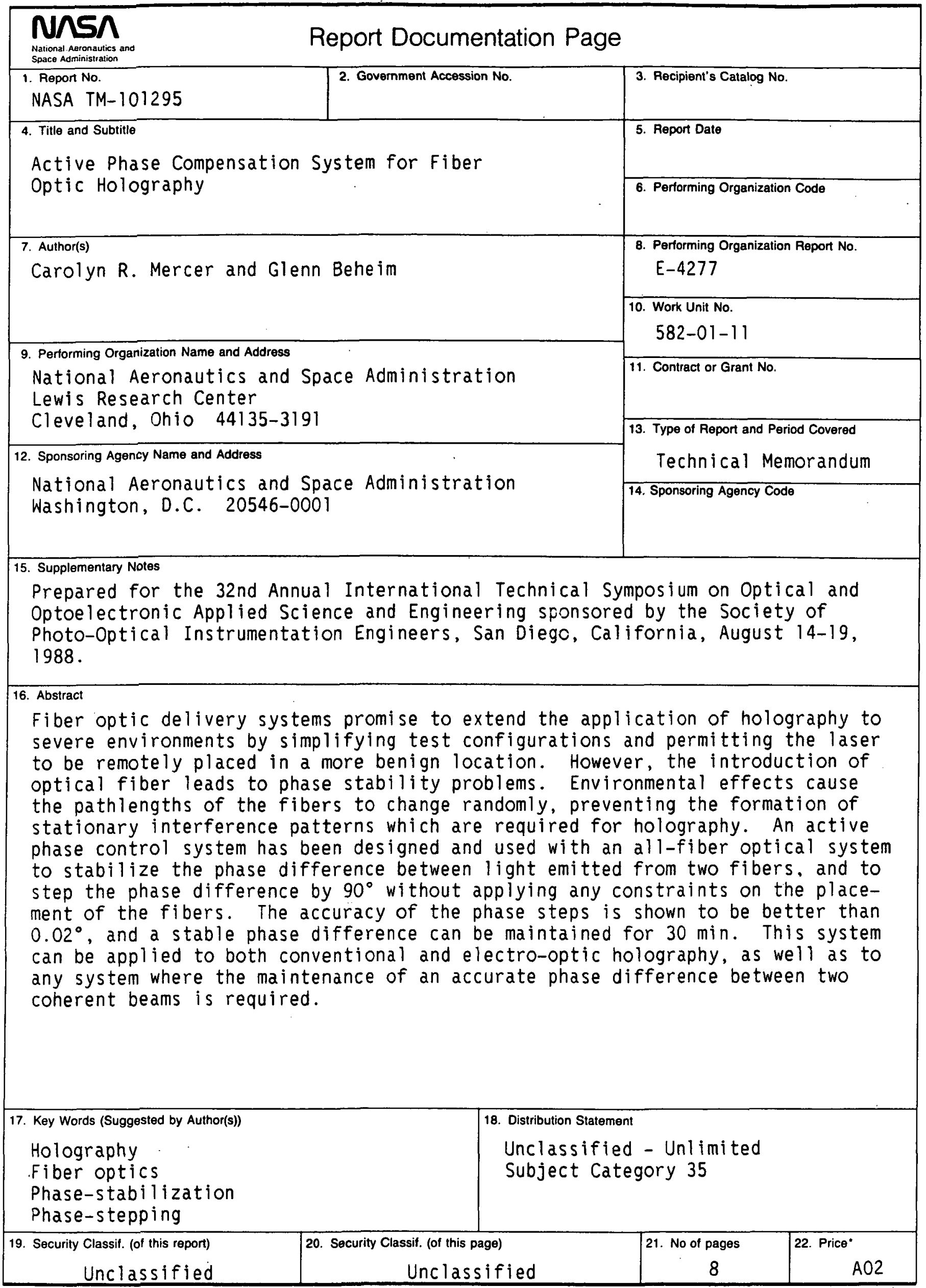


National Aeronautics and Space Administration

Lewis Research Center

Cleveland, Ohio 44135

Official Business

Penalty for Private Use $\mathbf{5 3 0 0}$

ADDRESS CORRECTION REQUESTED

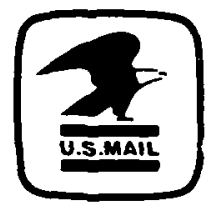

Postage and Fees Paid

National Aeronautics and

Space Administration

NASA 451 